\title{
Application of an Improved Granular Algorithm in C2 Design
}

\author{
Shuai Chen, Xuejun Ren, Wenjing Shao \\ Engineering University of CAPF, Xi'an, 710086, China
}

Keywords: command and control, granular algorithm, optimization.

\begin{abstract}
With the increasing scale of issue, the list planning performance remarkably decreases in the three-step method. To solve this issue, an improved granular algorithm is proposed in this paper for the C2 model design. At first, the adaptability measurement of model is optimized from the perspective of coarse granularity. Then, the completion period of task is optimized from the perspective of fine granularity. Thus, the two objectives can be optimized at the same time to some extent. According to case analysis and comparative test, this method can be used to simplify the complexity of solution and get ideal optimization results. Therefore, it is of good performance.
\end{abstract}

\section{Introduction}

Rapid development of information and network technology makes C2 organizations to be confronted with unprecedented challenges [1]. Due to limited competence of members in various organizations, complicated tasks and uncertain environment of task, future competitions between C2 organizations are more embodied in proper optimization and combination of individuals with limited competence, resources and information in those organizations as well as in the realization of organizational objectives by means of collaboration with members, allocation of resources and the optimal strategies.

The research team represented by Levchuk [2] proposed to use the three-step method for organizational structure. In this method, design issues about organizational structure are divided into three sub-optimization issues, so as to successively finish the allocation of task - platform, the distribution of platform - decision-making entity and the hierarchical relation between decision makers. This method is involved in two issues [3]: 1) with the increasing scale of issue, the performance of planning method in the first step will remarkably decrease; and 2) the optimization objective in the first step will have potential influence on optimization designs in the next two steps. In this paper, an improved granular algorithm is used to solve the two issues above.

\section{Adaptability measurement of organizational structure and task}

To prove the performance of the improved algorithm, the concept of adaptability measurement is introduced here. Adaptability measurement of organizational structure and task is used to measure an organization's performance in finishing relevant tasks. Two measurement parameters are mainly considered [4]: internal workload and external collaboration load of decision-making entity.

DM internal workload I (m) refers to accumulative load distributed to the platform of this decision-making entity:

$$
I(m)=\sum_{k=1}^{K} P D(k, m)
$$

DM external collaboration load E (m) refers to the collaboration sum of this decision-making entity and other decision-making entities:

$$
E(m)=\sum_{n=1, n \neq m}^{M} D(m, n)
$$

DM workload $\mathrm{W}(\mathrm{m})$ refers to the weighted sum of the two items above:

W1 and W2 are weights.

$$
W_{(m)}=W^{I} \cdot I_{(m)}+W^{E} \cdot E_{(m)}
$$


Adaptability measurement is the root-mean-square (RMS) of workloads of all decision-making entities.

$$
W_{R M S}(O, M)=\sqrt{\frac{1}{M} \sum_{m=1}^{M} W^{2}(m)}
$$

\section{Design method of organizational structure based on granular computation}

Stage I: This stage decides platforms and tasks for each decision-making entity. Objective is the minimum adaptability measurement of organizational structure and task.

Granulation issue of task set can be presented as follows:

$$
\min W_{R M S}(O, M)=\sqrt{\frac{1}{M} \sum_{m=1}^{M} W^{2}(m)}
$$

The greedy algorithm is used for solution, with specific steps listed as follows [5]:

Initial parameters: I (i) $=\left|G_{i}\right|, E(i)=0, i=1,2, \ldots, M ;|\cdot|$ stands for cardinal number of set;

Initial set: $\mathrm{T}^{1}$;

Step 1: take any value from $\mathrm{T}_{\mathrm{j}} \in \mathrm{T}^{1}$, choose the optimum allocation from $\mathrm{T}_{\mathrm{j}}$ to $\left\{D M_{i}\right\}$ to get the minimum value of the current $\mathrm{W}_{\mathrm{RMS}}(\mathrm{O}, \mathrm{M})$;

Step 2: make adjustment to relevant $E$ (i) according to the distribution results in Step 1;

Step 3: $T^{1} \leftarrow T^{1} /\left\{T_{j}\right\}$, if $T^{1} \neq \oint$, shift to Step 1 ; or, the algorithm will be terminated.

The genetic algorithm is used for granulation of platform set, with the flow diagram shown as follows:

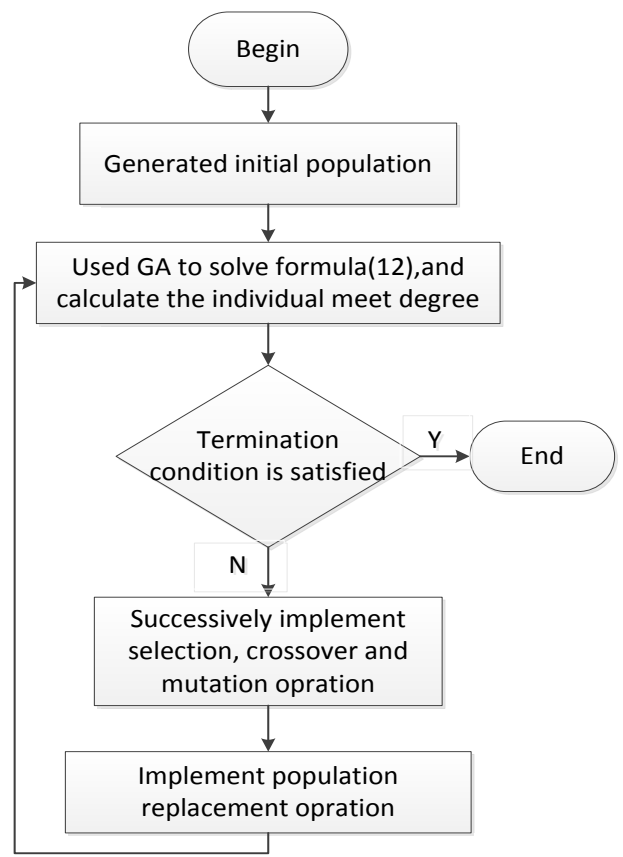

Fig.1: flow diagram

Stage II: This stage decides specific platform - task allocation controlled by each decisionmaking entity. Objective is the minimum completion period of task. The distribution from platform grain to task grain is obtained in Stage I. In this stage, the original planning issue is divided into some independent sub-planning issues.

Suppose that the decision-making entity $\mathrm{DM}_{\mathrm{m}}$ has $\mathrm{p}_{\mathrm{m}}$ platforms according to Stage $\mathrm{I}$, the platform set is expressed as $\mathrm{DM}_{\mathrm{m}}(\mathrm{P})$; the decision-making entity needs to finish $\mathrm{q}_{\mathrm{m}}$ tasks, the task set is expressed as $\mathrm{DM}_{\mathrm{m}}(\mathrm{T})$,

$$
\begin{gathered}
D M_{\mathrm{m}}(P)=\left\{P_{\mathrm{k}_{k}^{m}} \mid k_{k}^{m} \in\{1,2, \ldots, K\} h=1,2, \ldots, p_{m}\right\} \\
D M_{\mathrm{m}}(T)=\left\{T_{i_{j}^{m}} \mid i_{j}^{m} \in\{1,2, \ldots, N\} j=1,2, \ldots, q_{m}\right\}
\end{gathered}
$$


The capacity vector of $\mathrm{DM}_{\mathrm{m}}$ is expressed as $\mathrm{R}\left(\mathrm{DM}_{\mathrm{m}}\right)=\left[\bar{r}_{m 1}, \bar{r}_{m 2}, \ldots \bar{r}_{m 1}\right]$, $\bar{r}_{m l}$, indicating the quantity of function types controlled by this decision-making entity,

$$
\bar{r}_{m l}=\sum_{h=1}^{p_{m}} r_{k_{h}^{m} l}
$$

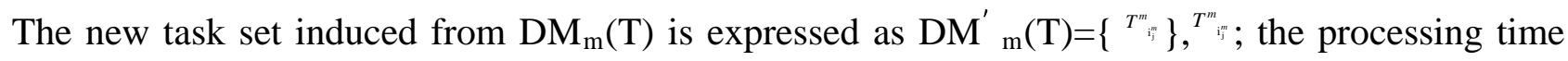
and the geographical limitation vector are estimated to be the same as ${ }^{T^{i p}}$; the resource demand

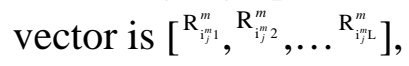

$$
\mathrm{R}_{i_{j}^{m} 1}^{m}=\min \left(\mathrm{R}_{\mathrm{i}_{j}^{m}{ }_{1}^{m}}, \bar{r}_{m l}\right)
$$

The priority relation of the new task set remains unchanged, namely:

Wherein, $\mathrm{j}_{1}, \mathrm{j}_{2}=1,2, \ldots, \mathrm{q}_{\mathrm{m}}$.

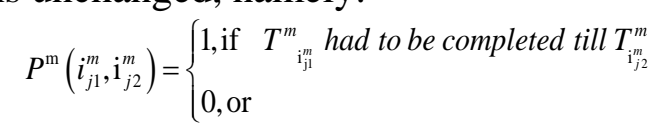

It is obvious from Formula (9) that the new task set $\mathrm{DM}^{\prime} \mathrm{m}(\mathrm{T})$ can be separately performed by decision-making entity $\mathrm{DM}_{\mathrm{m}}$.

For task set $\mathrm{DM}_{\mathrm{m}}^{\prime}(\mathrm{T})$ and platform set $\mathrm{DM}_{\mathrm{m}}(\mathrm{P})$, a reasonable task - platform allocation matrix $\mathrm{TP}^{\mathrm{m}}$ should meet the following conditions:

$$
\begin{gathered}
T P^{\mathrm{m}}\left(i_{j}^{m}, k_{h}^{m}\right)=\left\{\begin{array}{l}
1, \text { if } P_{\mathbf{k}_{h}^{m}} \quad \text { was allotted to } T_{\mathbf{i}_{j}^{m}}^{m} \\
0, \text { or }
\end{array}\right. \\
\sum_{h=1}^{p_{m}} T P^{m}\left(i_{j}^{m}, k_{h}^{m}\right) \cdot r_{k_{h}^{m} l} \geq R_{i_{j}^{m} l}^{m}
\end{gathered}
$$

Wherein, $\mathrm{j}=1,2, \ldots, \mathrm{q}_{\mathrm{m}} ; \mathrm{h}=1,2, \ldots, \mathrm{p}_{\mathrm{m}} ; \mathrm{m}=1,2, \ldots, \mathrm{M} ; \mathrm{l}=1,2, \ldots, \mathrm{L}$.

Theorem 1: suppose that there are $M$ reasonable task - platform allocation matrixes $\mathrm{TP}^{\mathrm{m}}, \mathrm{m}=1$, 2,..., M; then, the task - platform allocation matrix TP meeting Formula (13) is a reasonable allocation matrix:

Wherein: $\mathrm{i}=1,2, \ldots, \mathrm{N} ; \mathrm{k}=1,2, \ldots, \mathrm{K}$.

$$
T P^{\mathrm{m}}\left(i_{j}^{m}, k_{h}^{m}\right)=\left\{\begin{array}{l}
1, \text { if } P_{\mathrm{k}_{h}^{m}} \text { was allotted to } T_{\mathrm{i}_{j}^{m}}^{m} \\
0, \text { or }
\end{array}\right.
$$

Through the theorem, large-scale allocation tasks are divided into small-scale planning tasks. According to relevant researches, the improved MDLS algorithm [6] is of good performance when it is used for solution to small-scale planning issues. Therefore, this algorithm is used for solution. Specific processes are listed as follows:

Step 1: induce the new task set $\mathrm{DM}^{\prime}{ }_{\mathrm{m}}(\mathrm{T})$ from task set $\mathrm{DM}_{\mathrm{m}}(\mathrm{T})$;

Step 2: for task set $\mathrm{DM}^{\prime} \mathrm{m}(\mathrm{T})$ and platform set $\mathrm{DM}_{\mathrm{m}}(\mathrm{P})$, the improved MDLS algorithm is used to get task - platform allocation matrix $\mathrm{TP}^{\mathrm{m}}$;

Step 3: Formula (13) is used to calculate the final task - platform allocation matrix TP and to get the total time for completion of the task.

\section{Case analysis}

This case design a contingent of 14 communication teams handling 12 task missions, there are five decision-making entity, task flow shown in figure 2(task scenarios, communication resources and mission requirements specific data, please look up in the attached list). 


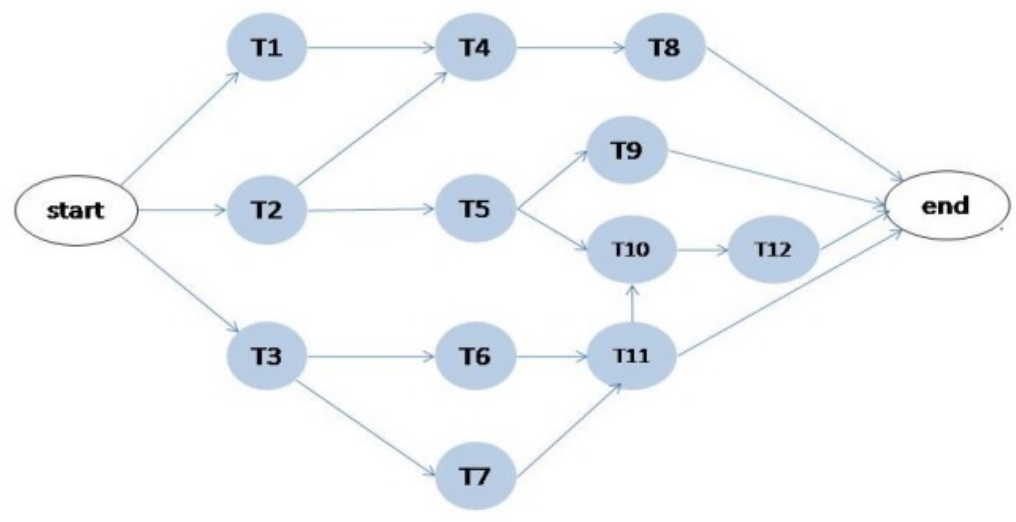

Fig.2: task flow

Stage 1: take 80 populations, take 380 evolution algebra, cross over rate from 0.6 , the mutation probability take 0.03 , through genetic algorithm, we can get decision-making entities and the contingent allocation and decision-making entities - task allocation results as shown in figure 3 :

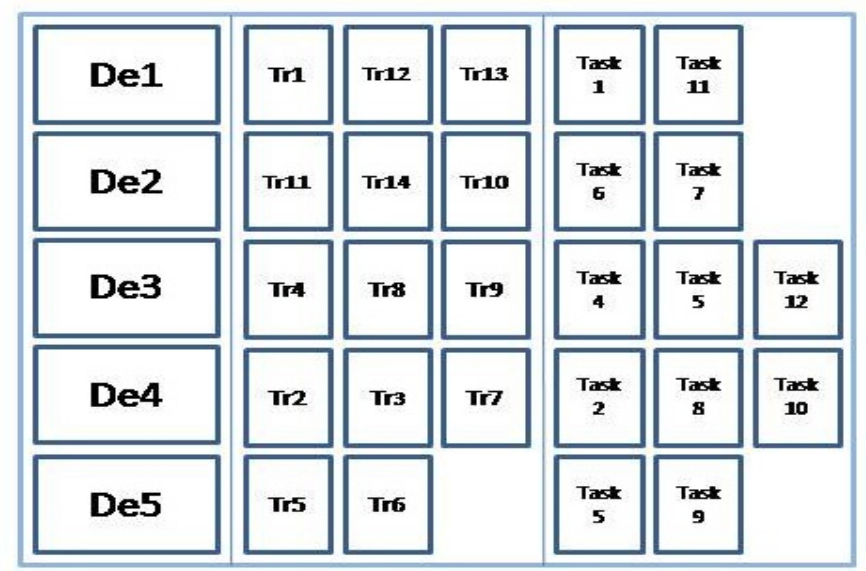

Fig.3: decision-making entities - task allocation results

Stage 2: for every decision entity's controlling resources and mission requirements, with the improved MDLS algorithm solving sub-planning problem [7], we get the final task - contingent allocation results as shown in figure 4 , the task completion time is 45 per unit time.

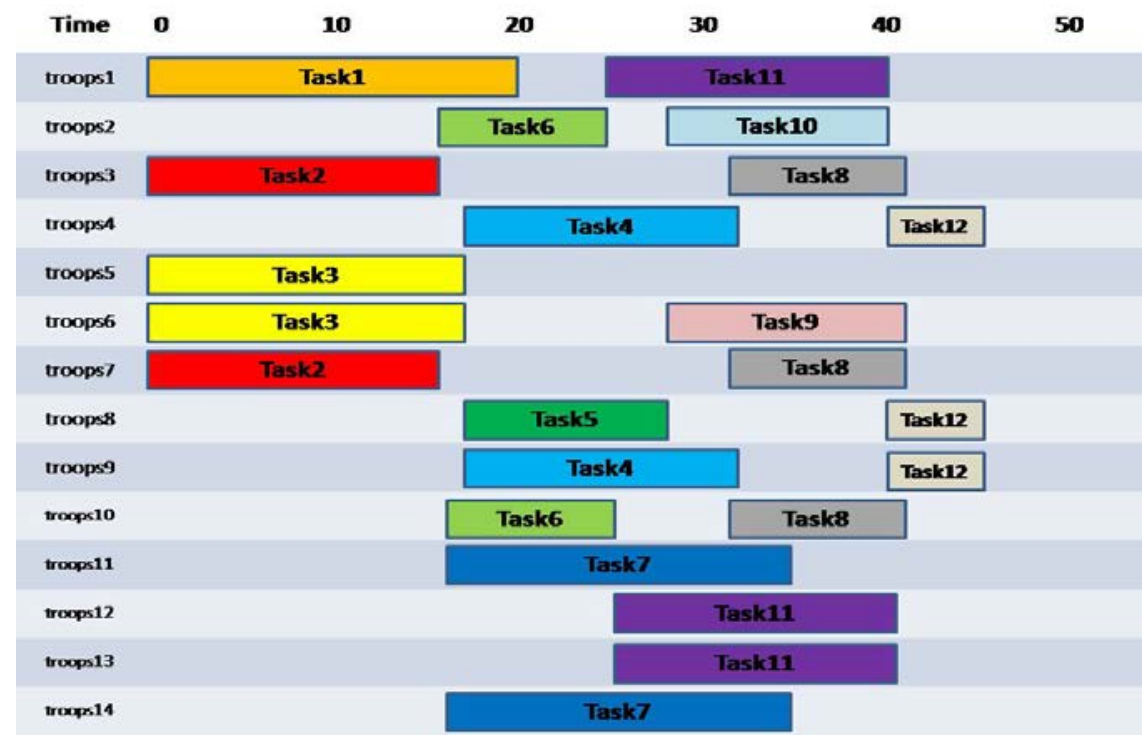

Fig.4: final task - contingent allocation results 


\section{The comparison}

Build the optimally organized structure $\mathrm{O} 1$ and $\mathrm{O} 2$ matching the mission $\mathrm{M}$ in two ways, and the results are shown below.

Table 1: Comparision

\begin{tabular}{|c|c|c|c|c|}
\hline \multirow{2}{*}{ mission } & \multicolumn{2}{|c|}{ O1 } & \multicolumn{2}{c|}{ O2 } \\
\cline { 2 - 5 } & $\begin{array}{c}\text { Adaptability } \\
\text { measurement }\end{array}$ & time & $\begin{array}{c}\text { Adaptability } \\
\text { measurement }\end{array}$ & time \\
\hline $\mathrm{M}$ & 4.05 & 46.6 & 7.18 & 98.9 \\
\hline
\end{tabular}

It can be seen that the method this thesis proposed is better than three-step method in this two indexes. Three-step method firstly optimizes the time the mission takes, and then optimizes the test of fitness. Through the case, the impact the former have on the latter is big.

\section{Conclusions}

Based on granular computation, the author proposes a new design method. Firstly, this method optimizes the adaptability measurement of tissue from the perspective of coarse granularity. Secondly, this method optimizes the completion period of mission from the perspective of fine granularity. Thus, two objectives can be optimized at the same time to some extent. It is foreseeable. Granular computation will play an important role in the settlement of complicated problems [8]. In subsequent researches, we will make further research on granulating method of task set.

\section{References}

[1] Q. Sun, D.S.Yang: Fire Control and Command Control. Vol. 18 (2013) No.12, p.1-5.

[2] B.X. Xiu, W.M. Zhang. Z.Liu and D.S.Yang: Systems Engineering and Electronics. Vol.29 (2007) No.7 p.1102-11087.

[3] X. Cao, J.Z. Xu: Information Command Control System and Simulation Technology. Vol. 27(2005) No.5, p.30-33.

[4] D.A. Zhou, D.G. Zhang and S.C. Chang: Command Control and Simulation, Vol. 30 (2008) No.4, p.13-17.

[5] D.S.Yang, W.M. Zhang, Z.Liu and C.Zhu: Systems Engineering Theory and Practice, Vol.32 (2005) No.5, p.83-88. .

[6] H.X. Liu: Design and adjust methodology of command and control organization(MS. National University of Defense Technology, China 2010),p.15

[7] D.S.Yang, W.M. Zhang, Z.Liu and C.Zhu: Armament Automation. VOl. 24(2005) No.2, p.18-21(In Chinese).

[8] D.L. Kleinman: International Command and Control Research and Technology Symposium, Washington, DC, June, 2003,32 